TITLE:

\title{
Viscoelastic effects on early stage of spinodal decomposition in dynamically asymmetric polymer blends
}

\section{$\operatorname{AUTHOR}(\mathrm{S}):$}

Takenaka, M; Takeno, H; Hashimoto, T; Nagao, M

\section{CITATION:}

Takenaka, M ...[et al]. Viscoelastic effects on early stage of spinodal decomposition in dynamically asymmetric polymer blends. JOURNAL OF CHEMICAL PHYSICS 2006, 124(10): 104904.

\section{ISSUE DATE:}

2006-03-14

URL:

http://hdl.handle.net/2433/39770

\section{RIGHT:}

Copyright 2006 American Institute of Physics. This article may be downloaded for personal use only. Any other use requires prior permission of the author and the American Institute of Physics. 


\title{
Viscoelastic effects on early stage of spinodal decomposition in dynamically asymmetric polymer blends
}

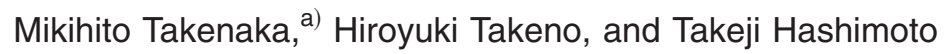 \\ Department of Polymer Chemistry, Graduate School of Engineering, Kyoto University, \\ Kyoto 606-8501, Japan \\ Michihiro Nagao \\ Institute for Solid State Physics, The University of Tokyo, Ibaraki 319-11, Japan
}

(Received 14 September 2005; accepted 10 November 2005; published online 10 March 2006)

\begin{abstract}
Spinodal decomposition induced by a rapid pressure change was investigated for a dynamically asymmetric polymer blend [deuterated polybutadiene (DPB)/polyisoprene (PI)] with a composition of 50/50 wt/wt by using time-resolved small angle neutron scattering. The time change in the scattered intensity distribution with wave number $(q)$ during the spinodal decomposition was found to be approximated by the Doi-Onuki theory [M. Doi and A. Onuki, J. Phys. II 2, 1631 (1992)]. The theoretical analysis yielded the $q$ dependence of the Onsager kinetic coefficient which is characterized by the $q^{-2}$ dependence at $q \xi_{\mathrm{ve}}>1$ with the characteristic length $\xi_{\mathrm{ve}}$ being much larger than the radius of gyration of DPB or PI. The estimated $\xi_{\mathrm{ve}}$ agrees well with that obtained previously in the relaxation processes induced by pressure change within the one phase region for the same blend. (c) 2006 American Institute of Physics. [DOI: 10.1063/1.2149861]
\end{abstract}

\section{INTRODUCTION}

The dynamics of phase separation processes have been widely investigated for various systems such as metal alloys, binary simple liquids, and polymer blends. ${ }^{1,2}$ Recently, it has been found that the dynamical asymmetry between constituent components in mixtures causes the coupling between stress and diffusion due to the asymmetric stress division. ${ }^{3}$ This coupling effect causes unique "viscoelastic effects" on the phase separation process, ${ }^{4}$ shear-induced concentration fluctuations or phase separation in polymer solutions, ${ }^{5}$ and non-single-exponential decay in dynamic light scattering (DLS) for polymer solutions. ${ }^{6}$ Theoretically, Doi and Onuki (DO) have taken this effect into account by formulating the time-dependent-Ginzburg-Landau (TDGL) type dynamical equation which incorporates the dynamical coupling between stress and diffusion. ${ }^{3}$ Onuki and Taniguchi further developed the linearized dynamical equation to apply to the early stage spinodal decomposition (SD). ${ }^{7}$

In the previous paper, ${ }^{8}$ we have investigated the relaxation processes of the concentration fluctuations induced by a rapid pressure change within the one phase region for a dynamically asymmetric deuterated polybutadiene (DPB)/ polyisoprene (PI) blend by using time-resolved small angle neutron scattering (SANS). The time change in the scattered intensity distribution with wave number $(q)$ during the relaxation processes was found to be approximated by the CahnHilliard-Cook (CHC) linearized theory. ${ }^{9}$ The CHC theory analysis yielded the $q$ dependence of the Onsager kinetic coefficient $\Lambda(q)=\Lambda(0)\left(1+q^{2} \xi_{\mathrm{ve}}^{2}\right)^{-1}$ which has the $q^{-2}$ dependence at $q \xi_{\mathrm{ve}}>1$ with $\xi_{\mathrm{ve}}$ being viscoelastic length. The $\xi_{\mathrm{ve}}$ value thus evaluated was much larger than the radii of gyra-

${ }^{a)}$ Electronic mail: takenaka@alloy.polym.kyoto-u.ac.jp tion of DPB and PI. The $\xi_{\text {ve }}$ value agrees with that predicted by the DO theory together with the fundamental parameters as obtained from viscoelastic data, indicating that the viscoelastic effects arising from the dynamical asymmetry between DPB and PI give rise to the $q$ dependence of $\Lambda(q)$ in the length scale of our observation, which is much larger than the radius of gyration $R_{g}$ or in the $q$ range of our observation which is much smaller than $1 / R_{g}$.

In this paper, we have investigated the time evolution of the concentration fluctuations after the onset of the pressure jump from the one phase region to spinodal region for the same DPB/PI blend with the one in the previous work by using time-resolved SANS. We shall test whether the DO theory is valid for the early stage spinodal decomposition as well as the relaxation processes of the concentration fluctuations within the one phase region and estimate the $q$ dependence of the Onsager kinetic coefficient.

\section{EXPERIMENTAL SECTION}

The DPB and PI used in this study were synthesized by living anionic polymerization. Table I shows the characterization of DPB and PI. In Table I, $M_{n}$ and $M_{w}$ designate number-averaged and weight-averaged molecular weights, respectively. The two polymers have different molecular weights and the dynamical properties, such as diffusion coefficient, are much different. As estimated in the previous paper, the ratio of the diffusion coefficient for PI to that of DPB is 34.8 . We studied a DPB/PI mixture with $50 / 50$ by weight. The DPB/PI blend has a lower critical solution temperature type and upper critical solution pressure type phase diagram and the spinodal temperature at $P=0.1 \mathrm{MPa}$ is $314.2 \mathrm{~K}^{8}$ We measured the spinodal decomposition processes at $318.5 \mathrm{~K}$ at $P=0.1 \mathrm{MPa}$. The quench depth $\varepsilon$ becomes $7.07 \times 10^{-3}$ where $\varepsilon$ is defined by 
TABLE I. Characterization of samples used in this study.

\begin{tabular}{|c|c|c|c|c|}
\hline $\begin{array}{l}\text { Sample } \\
\text { code }\end{array}$ & $M_{w}\left(\times 10^{-4}\right)$ & $M_{w} / M_{n}$ & $\begin{array}{l}\text { Molar volume } \\
\text { of monomer unit } \\
v^{\mathrm{a}}\left(\mathrm{cm}^{3} / \mathrm{mol}\right)\end{array}$ & $\begin{array}{c}\text { Unperturbed } \\
\text { radius of } \\
\text { gyration } R_{g}{ }^{\mathrm{b}}(\mathrm{nm})\end{array}$ \\
\hline DPB & $37.4^{\mathrm{c}}$ & $1.28^{\mathrm{c}}$ & 60.4 & 21.0 \\
\hline PI & $8.5^{\mathrm{d}}$ & $1.10^{\mathrm{d}}$ & 75.6 & 9.5 \\
\hline
\end{tabular}

${ }^{a} v=M_{0} / \rho$, where $M_{0}$ and $\rho$ are, respectively, the molecular weight of monomer and density.

${ }^{\mathrm{b}} R_{g}$ are obtained with unperturbed chain parameters from the viscoelastic measurement (Ref. 13). We used the unperturbed chain parameters of polybutadiene to estimate $R_{g}$ of DPB.

${ }^{\mathrm{c}}$ Determined with gel permeation chromatography with light scattering.

${ }^{\mathrm{d}}$ Determined with gel permeation chromatography calibrated by polyisoprene standard samples.

$$
\varepsilon \equiv \frac{\chi-\chi_{s}}{\chi_{s}}
$$

with $\chi$ and $\chi_{s}$ being the Flory-Huggins interaction parameter between DPB and PI per monomer unit and $\chi$ at spinodal point, respectively. The value of $\varepsilon$ for this experiment is estimated from the following temperature dependence of $\chi{ }^{8}$

$$
\chi=2.69 \times 10^{-3}-0.606 / T \text { at } 0.1 \mathrm{MPa} \text {. }
$$

The DPB/PI blend was dissolved into a homogeneous solution with toluene in which the total weight fraction of the polymers is 0.1 . The film specimen of the blend was obtained by evaporating the solvent slowly at room temperature. The film was further dried in vacuum for $24 \mathrm{~h}$ at room temperature and then molded into the disk with $1 \mathrm{~cm}$ diameter and $2 \mathrm{~mm}$ thickness for the SANS experiment. We installed the molded sample into the cell which is specially designed for the SANS measurement under high pressure (up to $200 \mathrm{MPa}$ ) and high temperature (up to $523 \mathrm{~K}$ ). The details of the cell for high pressure and temperature were described elsewhere. ${ }^{10}$ The SANS experiments were performed with SANS-U of the Institute for Solid State Physics of the University of Tokyo at JRR-3M reactor at Japan Atomic Energy Research Institute in Tokai. The neutron wavelength $\lambda$ used here was $0.8 \mathrm{~nm}$ and the sample-to-detector distance was $12 \mathrm{~m}$. All measured intensities were circularly averaged and calibrated into the absolute intensity $\left(\mathrm{cm}^{-1}\right.$ unit) with the incoherent scattering for Lupolene ${ }^{\circledR}$ after the correction of the electrical background scattering, the sample transmittance, and the scattering of an empty cell.

We took the following procedure to measure the time changes in the scattered intensity distribution induced by pressure change (hereafter defined as "quench") from 80.0 to $0.1 \mathrm{MPa}$ at $318.5 \mathrm{~K}$. The sample was first equilibrated at $318.5 \mathrm{~K}$ and $80.0 \mathrm{MPa}$ for $30 \mathrm{~min}$, where the mixture is in the one phase region, before the SANS measurement. The sample was then quenched from 80.0 to $0.1 \mathrm{MPa}$ at $318.5 \mathrm{~K}$, followed by a time-resolved SANS measurement as a function of time $t$ after the completion of the quench with data-acquisition time of $20 \mathrm{~s}$.

\section{RESULTS AND DISCUSSIONS}

Figure 1 shows the changes in the scattered intensity of the DPB/PI mixture with time after the onset of the quench

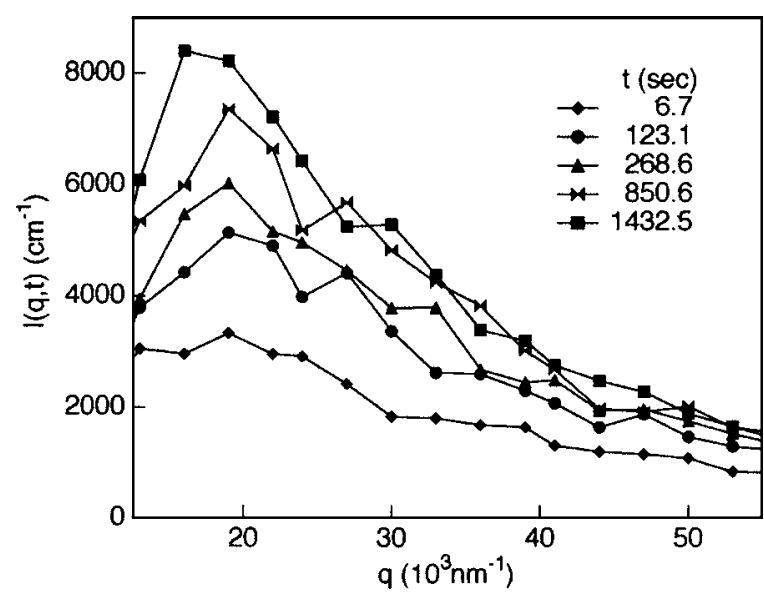

FIG. 1. Time change in SANS scattering function $I(q, t)$ after the pressure jump from 80.0 to $0.1 \mathrm{MPa}$ at $318.5 \mathrm{~K}$.

from 80.0 to $0.1 \mathrm{MPa}$ at $318.5 \mathrm{~K}$. In Fig. 1, after the quench, the scattering function $I(q, t)$ increases with time $t$ at observed $q$ region. The growth rate is larger at lower $q$ region.

In the previous paper, we found that the dynamics of the concentration fluctuations in the mixture after the onset of the quench within the one phase region can be well described by the DO theory. We shall check whether the DO theory can well describe the time change in the scattered intensity during the early stage spinodal decomposition. According to the DO theory, the linearized dynamical equation for the $q$-Fourier mode of the local concentration fluctuations of component DPB $\delta \phi_{\mathrm{DPB}}(q, t)$ in the DPB/PI binary mixture at $t$ is given by

$$
\frac{\partial}{\partial t} \delta \phi_{\mathrm{DPB}}(q, t)=-\frac{\Lambda_{\mathrm{eff}}(q) q^{2}}{S(q, \infty) / k_{B} T} \delta \phi_{\mathrm{DPB}}(q, t)+\varsigma(q, t) .
$$

Here $\Lambda_{\text {eff }}(q)$ is the $q$-dependent Onsager kinetic coefficient expressed by

$$
\Lambda_{\mathrm{eff}}(q)=\frac{\Lambda(0)}{1+q^{2} \xi_{\mathrm{ve}}^{2}} .
$$

$\xi_{\mathrm{ve}}$ is the viscoelastic length defined by

$$
\xi_{\mathrm{ve}}=\left(\frac{4}{3} \alpha \eta \Lambda(0)\right)^{1 / 2}
$$

where $\eta$ is the zero shear viscosity of the mixture and $\Lambda(0)$ is expressed by

$$
\Lambda(0)=\phi_{A} \phi_{B}\left(D_{A} N_{A} \phi_{B}+D_{B} N_{B} \phi_{A}\right) v_{0} / k_{B} T .
$$

$\alpha$ is a dynamical asymmetry parameter defined by

$$
\alpha=\frac{D_{A} N_{A}-D_{B} N_{B}}{D_{A} N_{A} \phi_{B}+D_{B} N_{B} \phi_{A}} .
$$

Here $D_{i}$ and $N_{i}$ are, respectively, the self-diffusion coefficient and the polymerization index of the $i$ th component $(i=A$ or $B$ in this case) in the blend. $S(q, \infty)$ is the so called virtual structure factor calculated by using the following equations based on the random phase approximation ${ }^{11}$ with $\chi$ at $P$ $=0.1 \mathrm{MPa}$ and $T=318.5 \mathrm{~K}$ : 


$$
\begin{aligned}
S_{\mathrm{eq}}(q, \infty)= & {\left[\frac{1}{\phi_{\mathrm{DPB}} N_{\mathrm{DPB}} v_{\mathrm{DBP}} S_{\mathrm{DPB}}(q)}+\frac{1}{\phi_{\mathrm{PI}} N_{\mathrm{PI}} v_{\mathrm{PI}} S_{\mathrm{PI}}(q)}\right.} \\
& \left.-\frac{2 \chi}{v_{0}}\right]^{-1},
\end{aligned}
$$

where $S_{i}(q)$ is the structure factor for the $i$ th component polymer $(i=\mathrm{DPB}$ or PI) given by

$$
S_{i}(q)=\frac{2}{x_{i}^{2}}\left[\left(\frac{h_{i}}{h_{i}+x_{i}}\right)^{h_{l}}-1+x_{i}\right],
$$

with

$$
x_{i}=q^{2} N_{n, i} b_{i}^{2} / 6
$$

and

$$
h_{i}=\left[\left(N_{w, i} / N_{n, i}\right)-1\right]^{-1} .
$$

$N_{n, i}$ and $N_{w, i}$ denote the number- and weight-averaged degrees of polymerization for the $i$ th component $(i=\mathrm{DPB}$ or $\mathrm{PI})$, respectively. $b_{i}$ and $v_{i}$ are, respectively, the statistical segment length and the molar volume of monomer unit, and $\phi_{i}$ the volume fraction for the $i$ th component. $v_{0}$ is the reference cell volume defined as $v_{0}=\left(\phi_{\mathrm{DPB}} / v_{\mathrm{DPB}}+\phi_{\mathrm{PI}} / v_{\mathrm{PI}}\right)^{-1}$. $\varsigma(q, t)$ is the random thermal force term as expressed by the following fluctuation-dissipation relation: $:^{2,12}$

$$
\left\langle\mathbf{s}(q, t) \mathbf{s}\left(q^{*}, t^{\prime}\right)\right\rangle=-2 k_{B} T \Lambda_{\text {eff }}(q) q^{2} \delta\left(t-t^{\prime}\right),
$$

where $k_{B}$ and $T$ are, respectively, the Boltzmann constant and absolute temperature and \langle\rangle denotes thermal average.

We can solve Eq. (3) and hence obtain the following time evolution of the scattering function $I(q, t)$ $\propto\left\langle\left|\delta \phi_{\mathrm{DPB}}(q, t)\right|^{2}\right\rangle$

$$
I(q, t)=I(q, \infty)+[I(q, 0)-I(q, \infty)] \exp [2 R(q) t],
$$

where $I(q, 0)$ and $R(q)$ are, respectively, $I(q, t)$ at $t=0$ and the relaxation rate of the $q$-Fourier modes of the concentration fluctuations. $R(q)$ is expressed by

$$
R(q)=-q^{2} \frac{\Lambda_{\mathrm{eff}}(q)}{\left[S(q, \infty) / k_{B} T\right]} .
$$

Rearranging Eq. (13), we obtain

$$
\log [|I(q, t)-I(q, \infty)|]=\log [I(q, 0)-I(q, \infty)]+2 R(q) t .
$$

Note that inside of logarithm in both sides should be absolute value, because $[I(q, t)-I(q, \infty)]$ or $[I(q, 0)-I(q, \infty)]$ becomes negative in the stable region of concentration fluctuations.

$I(q, \infty)$ is given by

$$
I(q, \infty)=k_{N} S(q, \infty),
$$

with

$$
k_{N}=N_{A}\left(\frac{a_{\mathrm{DPB}}}{v_{\mathrm{DPB}}}-\frac{a_{\mathrm{PI}}}{v_{\mathrm{PI}}}\right)^{2},
$$

where $a_{i}$ and $N_{A}$ are, respectively, the neutron scattering length of monomer unit for the $i$ th component and Avogadro's number. If the plots of $\log [|I(q, t)-I(q, \infty)|]$ versus $t$ show the linear relationship, we can estimate $R(q)$ from

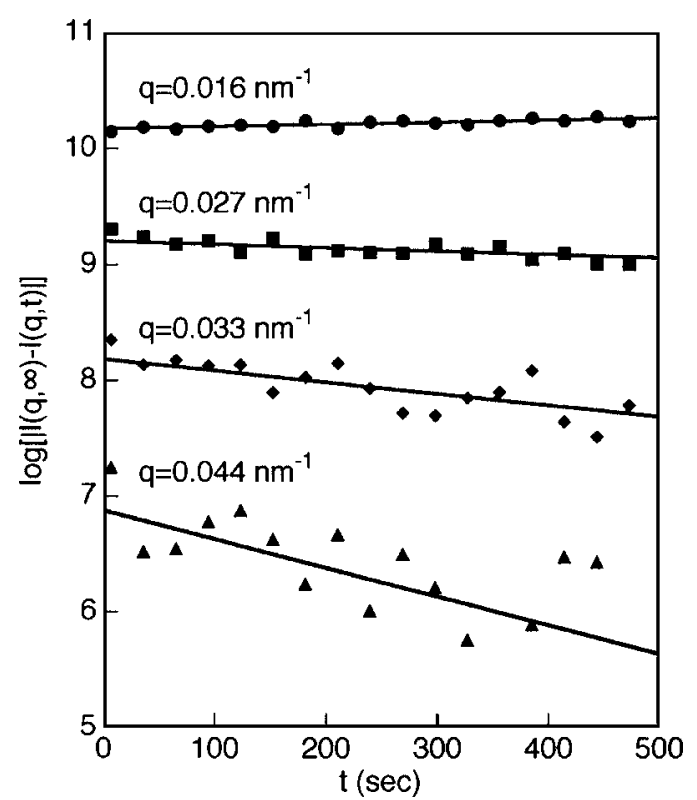

FIG. 2. $\log [I(q, t)-I(q, \infty)]$ plotted as function of time $t$ at $318.5 \mathrm{~K}$ and at fixed $q$ values. Solid lines were obtained by linear regression of data.

the slope of the plot where Log here denotes natural logarithms. In Fig. 2, $\log [|I(q, t)-I(q, \infty)|]$ is plotted as a function of $t$ after the onset of quench at $318.5 \mathrm{~K}$. The linearity between $\log [|I(q, t)-I(q, \infty)|]$ and $t$ can be found in each plot. We found that the slope of the plot becomes positive at $q<0.02 \mathrm{~nm}^{-1}$, since the thermodynamic force term $1 /\left[S(q, \infty) / k_{B} T\right]$ becomes negative due to the quench into unstable region.

Figure 3 shows the $q$ dependence of $R(q)$ estimated from the plots of $\log [I(q, t)-I(q, \infty)]$ versus $t$. $R(q)$ becomes positive at $q<0.02 \mathrm{~nm}^{-1}$ and decreases with $q$.

Figure 4 shows the $q$ dependence of the Onsager kinetic coefficient at $318.5 \mathrm{~K}$ (circles), as estimated from Eq. (14), i.e.,

$$
\Lambda_{\text {eff }}(q)=-\frac{R(q)\left[S(q, \infty) / k_{B} T\right]}{q^{2}} .
$$

Similar to the case in the relaxation process of the DPB/PI blend, the $q^{-2}$ behavior is found at higher $q$ region.

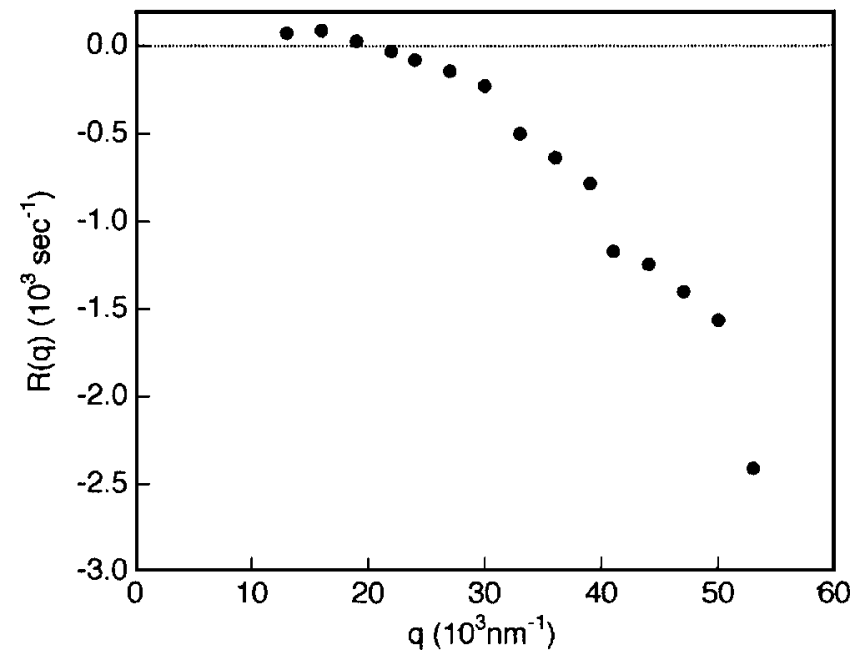

FIG. 3. Growth rate $R(q)$ for the $q$-Fourier mode of the concentration fluctuations plotted as a function of $q$ at $318.5 \mathrm{~K}$. 


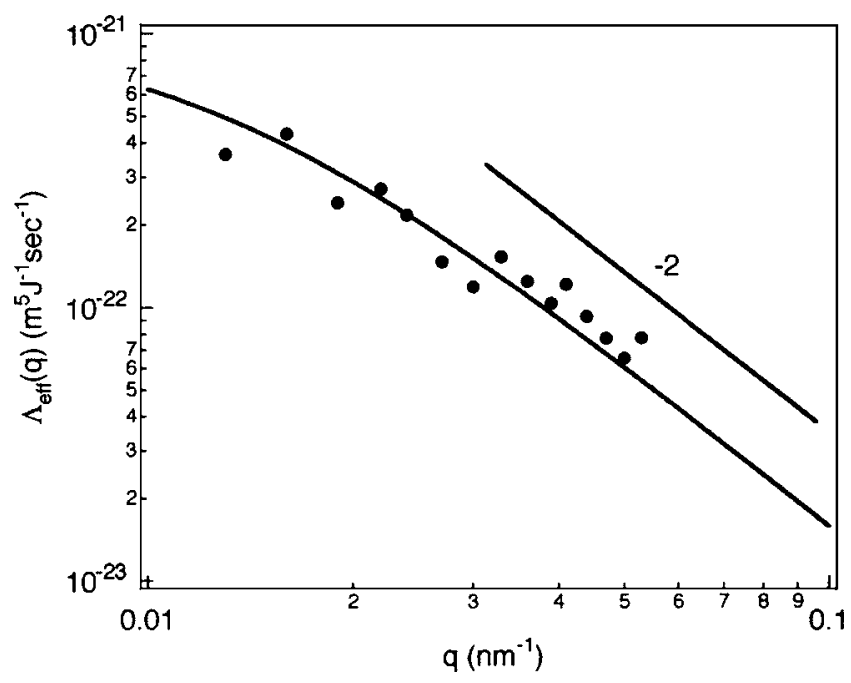

FIG. 4. $q$ dependence of the Onsager kinetic coefficient at $318.5 \mathrm{~K}$. The solid line indicates the fitting result with the DO theory [Eq. (4)].

We fitted the experimental results with Eq. (4) by using $\Lambda(0)$ and $\xi_{\mathrm{ve}}$ as adjustable parameters. The best-fitting result is displayed by the solid line in Fig. 4. The DO theory can well describe the experimental results on $\Lambda_{\text {eff }}(q)$. The estimated $\Lambda(0)$ and $\xi_{\mathrm{ve}}$ are $1.03 \pm 0.35 \times 10^{-21} \mathrm{~m}^{5} \mathrm{~J}^{-1} \mathrm{~s}^{-1}$ and $79.9 \pm 18.5 \mathrm{~nm}$, respectively. The obtained $\xi_{\mathrm{ve}}$ is much larger than $R_{g}$ of DPB and PI, which means that the Onsager kinetic coefficient can be well explained by the DO theory for dynamically asymmetric blends, not the Pincus theory for symmetric blends, ${ }^{14}$ as discussed in detail in the previous paper. Thus, the viscoelastic effects play a dominant role on the $q$ dependence of the Onsager kinetic coefficient in the case of the early stage spinodal decomposition as well as the relaxation process of the concentration fluctuations within the one phase region. As shown in our previous paper, we also obtained $\xi_{\mathrm{ve}}=64.6$ and $76.9 \mathrm{~nm}$ at 298.1 and $309.0 \mathrm{~K}$, respectively, in the experiment for the relaxation process after the onset of pressure jump within the one phase region. Figure 5 shows the temperature dependence of $\xi_{\mathrm{ve}}$. The $\xi_{\mathrm{ve}}$ estimated in this study agrees well with that obtained previously in the

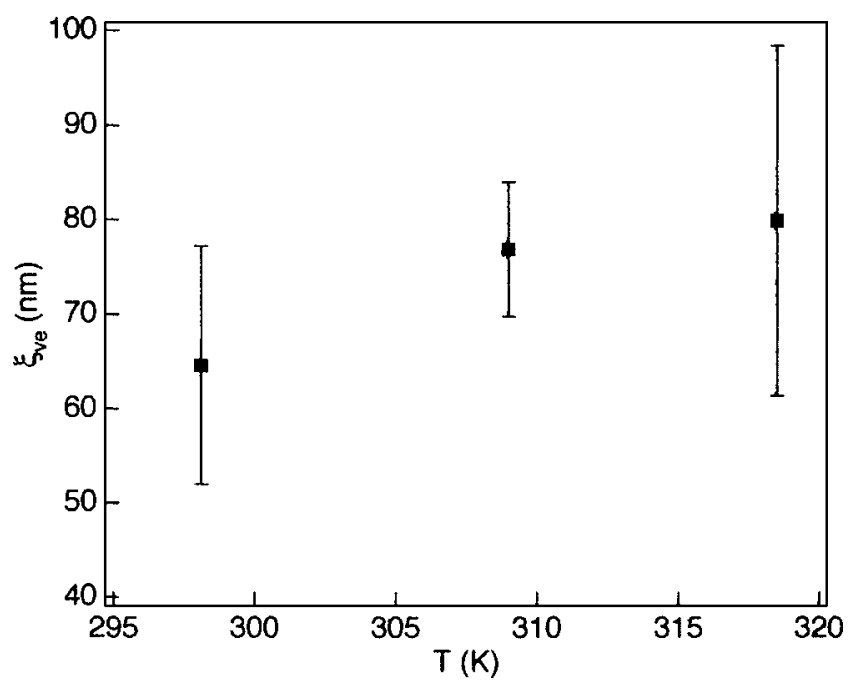

FIG. 5. Temperature dependence of viscoelastic length $\xi_{\text {ve }}$. relaxation processes induced by pressure change within the one phase region and the value of $\xi_{\text {ve }}$ slightly increases with temperature. According to the DO theory, if $\eta, \Lambda(0)$, and the tracer diffusion coefficient of both components have the same temperature dependence, $\xi_{\text {ve }}$ becomes independent of temperature [see Eqs. (5)-(7)]. The slight increase may be attributed to the fact that the tracer diffusion coefficient of each component has different temperature dependences.

\section{CONCLUSION}

We measured spinodal decomposition induced by a rapid pressure change for a dynamically asymmetric polymer blend [deuterated polybutadiene (DPB)/polyisoprene (PI)] with a composition of $50 / 50 \mathrm{wt} / \mathrm{wt}$ by using time-resolved small angle neutron scattering. We found that the time change in the scattered intensity during the spinodal decomposition can be approximated by the DO theory. The theoretical analysis yielded the $q$ dependence of the Onsager kinetic coefficient which is characterized by the $q^{-2}$ dependence at $q \xi_{\mathrm{ve}}>1$ with the characteristic length $\xi_{\mathrm{ve}}$ being much larger than the radius of gyration of DPB or PI. The estimated $\xi_{\text {ve }}$ agrees well with that obtained previously in the relaxation processes induced by pressure change within the one phase region for the same blend. The value of $\xi_{\mathrm{ve}}$ slightly increases with temperature. This is due to the fact that the tracer diffusion coefficient of each component has different temperature dependences.

\section{ACKNOWLEDGMENTS}

This work was supported in part by a Grant-in-Aid from Japan Society for the Promotion of Science (12640392, $13031053,15540392,16750182$, and 17105004) and by the Sumitomo Foundation.

${ }^{1}$ K. Binder, in Phase Transformations in Materials, edited by P. Haasen (VCH, Weinheim, 1990), Vol. 5, p. 405; T. Hashimoto, Phase Transitions 12, 47 (1988); in Structure and Properties of Polymers, edited by E. L. Thomas (VCH, Weinheim, 1993), p. 252.

${ }^{2}$ J. D. Gunton, M. S. Miguel, and P. S. Sahni, Phase Transitions 8, 269 (1983).

${ }^{3}$ M. Doi and A. Onuki, J. Phys. II 2, 1631 (1992).

${ }^{4}$ H. Tanaka, Phys. Rev. Lett. 71, 3158 (1993); J. Chem. Phys. 100, 5323 (1994); Phys. Rev. Lett. 76, 787 (1996); Phys. Rev. E 56, 4451 (1997); N. Toyoda, M. Takenaka, S. Saito, and T. Hashimoto, Polymer 42, 9193 (2001).

${ }^{5}$ P. K. Dixon, D. J. Pine, and X.-L. Wu, Phys. Rev. Lett. 68, 2239 (1992); T. Hashimoto and K. Fujioka, J. Phys. Soc. Jpn. 60, 356 (1991); T. Kume, T. Hattori, and T. Hashimoto, Macromolecules 30, 427 (1997); S. Saito and T. Hashimoto, J. Chem. Phys. 114, 10531 (2001); S. Saito, T. Hashimoto, I. Morfin, P. Lindner, and F. Boue, Macromolecules 35, 445 (2002); S. Saito, T. Hashimoto, I. Morfin, P. Lindner, F. Boue, and D. J. Pine, ibid. 36, 3745 (2003); S. Saito, S. Koizumi, K. Matsuzaka, S. Suehiro, and T. Hashimoto, ibid. 33, 2153 (2000); S. Saito, K. Matsuzaka, and T. Hashimoto, ibid. 32, 4879 (1999); S. Saito, A. Takenaka, N. Toyoda, and T. Hashimoto, ibid. 34, 6461 (2001).

${ }^{6}$ X.-L. Wu, D. J. Pine, and P. K. Dixon, Phys. Rev. Lett. 66, 2408 (1991).

${ }^{7}$ A. Onuki and T. Taniguchi, J. Chem. Phys. 106, 5761 (1997).

${ }^{8}$ M. Takenaka, H. Takeno, H. Hasegawa, S. Saito, T. Hashimoto, and M. Nagao, Phys. Rev. E 65, 021806 (2002).

${ }^{9}$ J. W. Cahn, J. Chem. Phys. 42, 93 (1965); H. E. Cook, Acta Metall. 18, 297 (1970).

${ }^{10}$ H. Takeno, M. Nagao, Y. Nakayama, H. Hasegawa, T. Hashimoto, H. Seto, and M. Imai, Polym. J. (Tokyo, Jpn.) 29, 931 (1997).

${ }^{11}$ P. G. de Gennes, Scaling Concepts in Polymer Physics (Cornell Univer- 
sity Press, Ithaca, 1979); S. Sakurai, T. Izumitani, H. Hasegawa, T. Hashimoto, and C. C. Han, Macromolecules 24, 4844 (1991); S. Sakurai, K.

Mori, A. Okawara, K. Kimishima, and T. Hashimoto, ibid. 25, 2679 (1992).
${ }^{12}$ K. Binder, J. Chem. Phys. 79, 6387 (1983).

${ }^{13}$ J. Mays, N. Hadjichristidis, and L. J. Fetters, Macromolecules 17, 2723 (1984).

${ }^{14}$ P. Pincus, J. Chem. Phys. 75, 1996 (1981). 